\title{
Impact energy attenuation performance of football headgear
}

Andrew S McIntosh, Paul McCrory

\begin{abstract}
Objectives-Commercially available football head protectors were tested to determine their impact energy attenuation performance and ability to reduce the likelihood of concussion.

Methods-Prospective study using standardised impact test methods with both rigid (magnesium) and Hybrid III headforms.

Results-Eight commercially available head protectors from six manufacturers were tested. The magnitude of the headform accelerations increased as the drop height was increased, ranging from a minimum of $64 \mathrm{~g}$ from a height of $0.2 \mathrm{~m}$ to a maximum of $1132 \mathrm{~g}$ from a height of 0.6 $m$. The head injury criterion and maximum headform acceleration values followed a similar trend. A steep increase was noted in the magnitude of maximum headform acceleration and head injury criterion when the drop height was increased from 0.4 to $0.5 \mathrm{~m}$. This indicates that the foam material was completely compressed at an impact energy above about $20 \mathrm{~J}$ and therefore offers little protection against impacts of greater severity. Repeated tests using a drop height of $0.3 \mathrm{~m}$ showed that some helmets exhibit a "memory" effect, whereby impact performance is reduced by up to $50 \%$ with repeated impacts.
\end{abstract}

Conclusions-Laboratory tests indicate that current commercially available football headgear performance will not reduce the likelihood of concussion. The absence of internationally recognised standards for soft headgear designed to ameliorate concussion is a major deficiency in sports injury prevention.

(Br F Sports Med 2000;34:337-341)

Keywords: football; headgear; helmet; head; concussion

Melbourne,

Department of

Neurology, Austin and

Repatriation Medical

Centre, Heidelberg,

Victoria, Australia

3084 and Olympic Park

Sports Medicine

Centre, Swan Street,

Melbourne, Australia

3004

P McCrory

Correspondence to: Dr A McIntosh

email:

a.mcintosh@unsw.edu.au

Accepted for publication 10 March 2000 decreasing the magnitude of the impact force to the head. This in turn leads to a decrease in head acceleration and less potentially injurious stress on the brain and cerebrovascular tissues. The means by which helmets attenuate energy is through liner deformation, so that the magnitude of the impact force is inversely proportional to the magnitude of the liner deformation. ${ }^{2}$ Although "soft" helmets or headgear are widely available and often used in football, little is known about their actual performance during impact. ${ }^{3}$ The degree of protection offered by headgear is contentious, with some people advocating that their function should be to prevent abrasions and lacerations ${ }^{34}$ and others advocating that their role should be to reduce concussion.

Headgear wearing rates or controlled studies of headgear effectiveness have not been well documented. Gerrard et $a \bar{l}$ reported that $20 \%$ of rugby union players used headgear in 1992 . Our experience in studying headgear use in under 15 rugby union in 1999 indicates that the voluntary wearing rate in this age group is about $60 \%$. Headgear wearing rates probably differ between codes, age groups, and skill levels. The potential for headgear to reduce rates of concussion relies not only on players wearing it, but also on the ability of the headgear to attenuate impact energy. ${ }^{2}$

In this study, a selection of commercially available football headgear was tested to determine impact energy attenuation performance with regard to the ability to reduce likelihood of concussion and more severe head injury.

\section{Methods}

Eight soft helmets from six manufacturers were tested using a standardised methodology. Owing to the lack of availability of two models at the time of testing, a limited number of tests were performed on these two models, whereas all tests were performed on the remaining six medium sizes. Tests were conducted on five small sizes selected from the best performing models in the medium size tests. Tests were conducted using two separate headforms, a rigid magnesium alloy headform and a Hybrid III headform.

RIGID HEADFORM TEST

A standard test methodology was used as per the 1999 Australian standard AS/NZS 2512.1.1. ${ }^{6}$ This is similar to many international helmet testing standards such as the SNELL and ANSI standards. The impact energy attenuation test is similar to the 1998 standard for headgear of the International Rugby Board (IRB), ${ }^{7}$ except that the latter does not specify

Table 1 Velocities and impact energies of test matrix protocol 
Table 2 Summary of rigid headform test results

\begin{tabular}{|c|c|c|c|c|c|c|c|c|}
\hline \multirow[b]{2}{*}{ Size } & \multirow[b]{2}{*}{ Impact site } & \multirow[b]{2}{*}{ Condition } & \multirow{2}{*}{$\begin{array}{l}\text { Drop } \\
\text { height } \\
(\mathrm{m})\end{array}$} & \multirow{2}{*}{$\begin{array}{l}\text { No of } \\
\text { models } \\
\text { tested }\end{array}$} & \multicolumn{2}{|c|}{$\begin{array}{l}\text { Maximum } \\
\text { headform } \\
\text { acceleration (g) }\end{array}$} & \multicolumn{2}{|c|}{$\begin{array}{l}\text { Head injury } \\
\text { criterion }\end{array}$} \\
\hline & & & & & Min & $\operatorname{Max}$ & Min & $\operatorname{Max}$ \\
\hline \multirow[t]{10}{*}{ Medium } & Fronto-lateral & Ambient & 0.2 & 6 & 64 & 483 & 73 & 1192 \\
\hline & & & 0.3 & 8 & 106 & 569 & 183 & 2106 \\
\hline & & & 0.4 & 6 & 141 & 832 & 279 & 4132 \\
\hline & & & 0.5 & 6 & 482 & 1073 & 1451 & 6000 \\
\hline & & & 0.6 & 8 & 524 & 1132 & 2225 & 7253 \\
\hline & & Hot & 0.3 & 7 & 109 & 689 & 184 & 2850 \\
\hline & & & 0.6 & 7 & 547 & 1032 & 2191 & 7089 \\
\hline & & Cold & 0.3 & 7 & 108 & 672 & 183 & 2523 \\
\hline & & & 0.6 & 7 & 443 & 1085 & 1458 & 6620 \\
\hline & Centre-front & Ambient & 0.4 & 6 & 819 & 1026 & 4584 & 6118 \\
\hline \multirow[t]{2}{*}{ Small } & Fronto-lateral & Ambient & 0.2 & 5 & 68 & 105 & 84 & 148 \\
\hline & & & 0.3 & 5 & 97 & 298 & 186 & 713 \\
\hline
\end{tabular}

the drop mass, the filter characteristics for headform acceleration, or sampling rate. These are critical parameters in impact testing. ${ }^{8}$ In addition, the drop height is limited to $300 \mathrm{~mm}$. All testing was performed at the New South Wales Roads and Traffic Authority's "Crashlab" in Sydney, which is certified by the National Association for Testing Authorities and routinely conducts test to AS/NZS 2512.1.1.

A size C magnesium alloy headform was used to test the medium size headgear. The total drop mass was $5 \mathrm{~kg}$. A size B magnesium alloy headform was used to test small size headgear. The total drop mass was $4.1 \mathrm{~kg}$. A single impact was delivered to one site as discussed below. Headform acceleration was sampled at $10 \mathrm{kHz}$ with an Endevco 7265 accelerometer. Acceleration data were filtered using a Channel Class 1000 filter, and impact velocity was measured using a light gate. Tests were conducted on headgear conditioned to cold $\left(-10^{\circ} \mathrm{C}\right)$, ambient $\left(20^{\circ} \mathrm{C}\right)$, and hot $\left(50^{\circ} \mathrm{C}\right)$ environmental conditions according to AS/ NZS 2512.2 (1998). ${ }^{9}$

As well as the single impact testing above, further testing to determine impact performance under repeated frontal/lateral impacts was performed, with impacts at three minute intervals to a total of five times. For single impacts, only one impact per test site was delivered.

HYBRID III HEADFORM TEST

A second series of tests were conducted on the same selection of headgear to determine the impact energy attenuation performance using a Hybrid III headform. This headform is used in motor vehicle crashworthiness testing and has a deformable skin that more closely resembles a human head than the rigid headform. All tests were conducted at the University of New South Wales, School of Safety Science, Biomechanics and Gait Laboratory using a standard drop test rig. The Hybrid III 50th percentile headform and instrumentation had a total drop mass of $4.7 \mathrm{~kg}$. Three orthogonal Endevco 7264 accelerometers were used to measure headform acceleration at the headform's centre of mass. Headform acceleration was sampled at $10 \mathrm{kHz}$ and filtered using a Channel Class 1000 filter. Tests were conducted on headgear at an ambient temperature and using the same impact sites as for the rigid headform. Comparative tests between

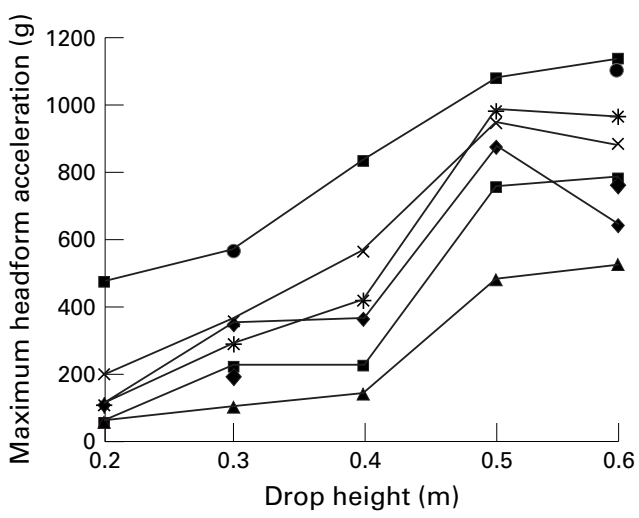

Figure 1 Maximum headform acceleration $v$ drop height for eight models of rigid headform. Complete results are given for six models, and results for the 0.3 and $0.6 \mathrm{~m}$ tests only for the other two.

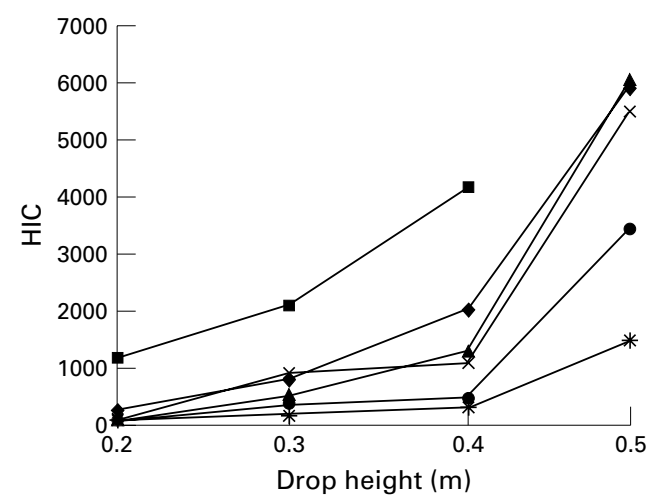

Figure 2 Head injury criterion (HIC) $v$ drop height for six models of rigid headform. The results for two models were omitted to keep the scale in proportion with an HIC of 1000 as the standard pass/fail criterion.

the rigid and deformable headforms were performed using pieces of polymeric foam cut from the same samples.

SELECTION OF ANATOMICAL TEST IMPACT SITES On the basis of previously published studies in which video analysis of head impacts in rugby league, rugby union, and Australian football were performed, the temporal-parietal area was identified as the most frequently struck area and thus selected for testing purposes. ${ }^{10}{ }^{11}$ In addition, because published experimental animal research has suggested that fronto-lateral impacts may also result in brain shearing injury, this impact site was also tested..$^{12}$

SELECTION OF IMPACT ENERGY AND VELOCITY

Tests were conducted over a range of impact speeds and energies. These were selected on the basis of initial performance of the headgear and the standard test protocols. It was found that performance was poor from drop heights of greater than $0.6 \mathrm{~m}$, so further increments in drop height were not performed. Table 1 gives the impact velocities and energies for the various drop heights.

\section{Results}

RIGID HEADFORM TESTS: IMPACT ENERGY ATTENUATION PERFORMANCE

A total of 78 rigid headform single impact tests were performed. When sufficient samples were available at the time of testing, one of each 


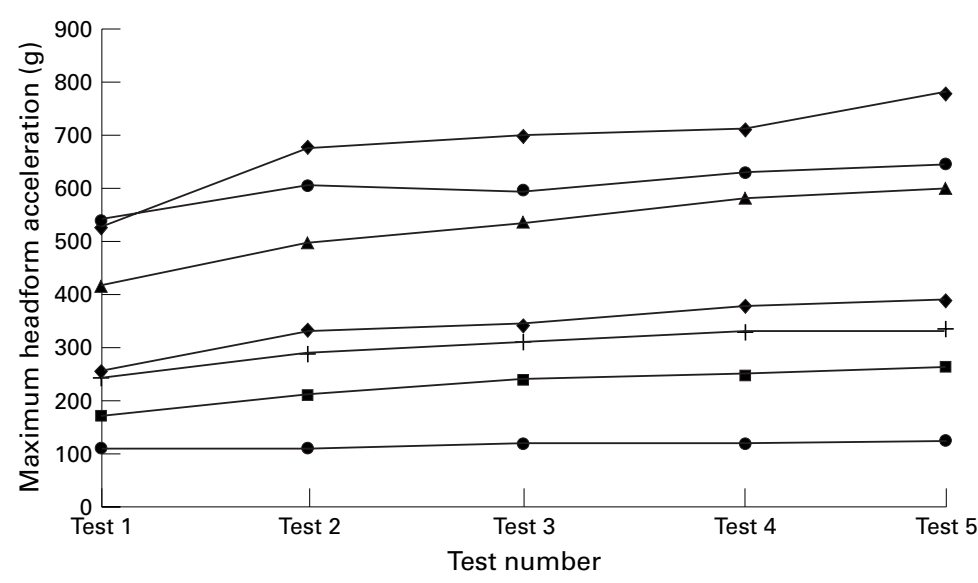

Figure 3 Maximum headform acceleration for repeated tests from a drop height of $0.3 \mathrm{~m}$ for seven models. range of headform acceleration maxima and HIC were greater-for example, $483 \mathrm{~g}$ compared with 689 and $672 \mathrm{~g}$ for $0.3 \mathrm{~m}$ drops under ambient, hot, and cold conditions respectively.

A second common impact site, centre front, was assessed on six of the models from a drop height of $0.4 \mathrm{~m}$. The lowest maximum headform acceleration at this site was $819 \mathrm{~g}$ compared with $141 \mathrm{~g}$ for the fronto-lateral impacts.

There was a steep increase in the magnitude of maximum headform acceleration and HIC when the drop height was increased from 0.4 to $0.5 \mathrm{~m}$. This indicates that the foam material was completely compressed at impact energies above about $20 \mathrm{~J}$.

Repeated tests were conducted from a drop height of $0.3 \mathrm{~m}$ using seven models. Thirty five tests were conducted. They showed that the greatest change in headform acceleration over the five tests was $244 \mathrm{~g}$ (about $50 \%$ of the initial acceleration) and the least change $7 \mathrm{~g}$ (about $5 \%$ of the original value). Figure 3 shows the test results. figs 1 and 2 show the results. Tab indicates how many different models were tested under each condition and presents the results of maximum headform acceleration and the head injury criterion $\left(\mathrm{HIC}_{36}\right)$. The minimum and maximum values represent the range of headform acceleration maxima and HIC for all models tested under the defined conditions, and reflect the best and worst performance respectively. HIC and maximum headform acceleration results followed a similar trend as they are related, but $\mathrm{HIC}_{36}$ is sensitive to both the magnitude and duration of headform acceleration.

There was a large range of maximum headform accelerations and HIC values between models at each drop height. As expected, the magnitude of the headform accelerations and HIC increased as the drop height was increased. At a drop height of $0.2 \mathrm{~m}$, the smallest maximum headform acceleration was $64 \mathrm{~g}$ for the six models tested. Even at this low drop height and low energy, one model gave a result of $483 \mathrm{~g}$. Under the most severe test conditions (the $0.6 \mathrm{~m}$ test) the best result - that is, lowest headform acceleration-was $524 \mathrm{~g}$ compared with the worst at $1132 \mathrm{~g}$. Figures 1 and 2 show the results for each model. It can be observed that the models tended to be ranked in the same order under all impact conditions.

Tests were undertaken with the headgear conditioned to hot and cold to determine whether the environmental conditions affected performance. The results were similar to those obtained at ambient temperature, except that, at lower impact severities, the higher end of the
HYBRID III HEADFORM: IMPACT ENERGY

ATTENUATION PERFORMANCE

The impact speeds were held constant in this test protocol, and, because of the different drop masses, the impact energies for each drop height are slightly different (table 1 ). The comparison of headform acceleration for identical samples of helmet foam show that the results are affected dramatically by the headform. From the $0.5 \mathrm{~m}$ drop height, the acceleration for the rigid headform was about four times greater than for the Hybrid III with the polyethylene foam and about six times greater with the composite foam. At the lower drop height, the differences were not as pronounced, probably because further foam deformation was still available. As the Hybrid III has a deformable "skin", the thickness of the compressible layers was almost double that of the rigid headform tests. Table 3 gives the results.

\section{Discussion}

Head injury and concussion are common to all codes of football. In prospective studies, the concussion rate in Australian football is 4.7 per 1000 player hours. ${ }^{1}$ In rugby union and league, prospective injury data are limited; however, the concussion rate is thought to be about three per 1000 player hours in rugby union and eight per 1000 player hours in league. ${ }^{1}$ A possible method of reducing head injury incidence and severity is for players to wear headgear, also

Table 3 Results summary using Hybrid III headform with helmet and foam testing: a comparison of headform accelerations for the matched headgear sample is provided

\begin{tabular}{|c|c|c|c|c|c|c|c|c|}
\hline \multirow[b]{2}{*}{ Size/type } & \multirow[b]{2}{*}{ Impact site } & \multirow[b]{2}{*}{ Condition } & \multirow{2}{*}{$\begin{array}{l}\text { No of } \\
\text { models } \\
\text { tested }\end{array}$} & \multirow{2}{*}{$\begin{array}{l}\text { Drop } \\
\text { height } \\
\text { (m) }\end{array}$} & \multicolumn{2}{|c|}{$\begin{array}{l}\text { Maximum } \\
\text { headform } \\
\text { acceleration } \\
\text { Hybrid III (g) }\end{array}$} & \multicolumn{2}{|c|}{$\begin{array}{l}\text { Maximum } \\
\text { headform } \\
\text { acceleration } \\
\text { rigid }(\mathrm{g})\end{array}$} \\
\hline & & & & & Min & Max & Min & $\operatorname{Max}$ \\
\hline \multirow[t]{2}{*}{ Helmet test - medium } & \multirow[t]{2}{*}{ Fronto-lateral } & \multirow[t]{2}{*}{ Ambient } & 5 & 0.3 & 80 & 106 & 106 & 363 \\
\hline & & & 5 & 0.6 & 153 & 282 & 524 & 960 \\
\hline \multirow[t]{2}{*}{ Foam tests - polyethylene $33 \mathrm{~kg} / \mathrm{m}^{3}, 12 \mathrm{~mm}$ thick } & \multirow[t]{2}{*}{ Fronto-lateral } & \multirow[t]{2}{*}{ Ambient } & 1 & 0.3 & & 81 & & 111 \\
\hline & & & 1 & 0.5 & & 133 & & 501 \\
\hline \multirow[t]{2}{*}{ Foam tests - composite } & \multirow{2}{*}{ Fronto-lateral } & \multirow{2}{*}{ Ambient } & 1 & 0.3 & & 65 & & 139 \\
\hline & & & 1 & 0.5 & & 133 & & 884 \\
\hline
\end{tabular}


known as head protectors or headguards. In the rugby codes and Australian football, lightweight soft shell head protectors are used, in contrast with the hard shell helmets used in American Football.

Overall headgear wearing rates are modest in rugby union, ${ }^{5}$ league, and Australian football. For wearing rates to increase, participants need to expect that headgear will afford definite protection. Anecdotal evidence indicates that junior players, often as the result of parental pressure, wear headgear to prevent concussion, whereas senior players have fewer expectations about the prevention of concussion, but use the headgear for protection against superficial injuries and to prevent traumatic headaches rather than brain injury. There is also a concern that young players who wear headgear may change their playing behaviour and paradoxically effect an increase in head injuries because of misguided faith in an ineffective device.

The mechanics of head injury have been extensively studied using mathematical, experimental, and observational methods. ${ }^{14-16}$ Studies of head injuries have arisen primarily out of the need to provide protection for road users rather than for sport. There are obvious difficulties in extrapolating the results from motor vehicle accident injuries to football players. However, the basic biomechanical injury mechanisms are the same, namely forces applied directly to the head or through the trunk and neck accelerate the head producing internal stresses within the brain, which in turn may result in pathological injury. The resultant brain injury is related to the magnitude of the impact force, the location of impact, and the resultant head and brain acceleration.

$\mathrm{Head} /$ headform acceleration and its derivative the $\mathrm{HIC}_{36}$ are used to assess the performance of safety systems including helmets. At present, the tolerance limit for headform acceleration as reflected in international standards is between 200 and $300 \mathrm{~g}$ - for example, CAN/ CSA-D113.2-M89 and AS/NZS 2063:1996, both pedal cycle helmet standards. It can be argued that a more realistic tolerance limit would be as low as $150 \mathrm{~g} .{ }^{18}{ }^{18}$ The threshold for concussion for HIC is 1000 , and there is evidence that this may be too high for safety. ${ }^{11} 18$

The results show that, on the basis of existing head injury models, all of the currently available commercial headgear have very limited ability to reduce concussion. There are also significant performance differences between brands and models, and even between models manufactured by the same companies. Even if the more generous injury thresholds of $300 \mathrm{~g}$ and a HIC of 1000 are applied to the data, all headgear tested loses any protective capacity once the impact energy is greater than $20 \mathrm{~J}$. As can be seen in figs 1 and 2, these thresholds have been exceeded by almost all models above impact energies of about $15 \mathrm{~J}$. If injury thresholds are reduced-for example, maximum headform acceleration of $200 \mathrm{~g}$ and HIC of 800 - then the performance is seen to be even poorer, with most headgear tested providing insufficient protection for impacts of greater than $10 \mathrm{~J}$. A $10 \mathrm{~J}$ impact is equivalent to a head impact from a drop height of $20 \mathrm{~cm}$ or 2 $\mathrm{m} / \mathrm{s}$.

The test results for repeated impacts on the same sample headgear showed that the foam in some models exhibits a memory effect - that is, after each successive impact the impact energy attenuation performance is reduced. This indicates that the foam does not return to its original thickness. As football headgear is designed to perform under repeated impacts, this is an important function that also needs to be assessed in any standard.

If the Hybrid III headform tests are considered a more realistic reflection of the impact situation, because of the headform's biofidelity, then the safety performance of the headgear is extended to around $28 \mathrm{~J}$. However, in this analysis, the deformation of the ground or other bodies has been accounted for by the headform. When these results are extrapolated to real impacts, they still indicate that the ability of headgear to reduce the likelihood of concussion is low. The use of Hybrid III headforms is unusual in helmet testing. The results for the rigid headform are presented as the "standard" results.

With regard to the IRB specifications, only four out of the eight models would have passed the impact energy attenuation test requirements for the IRB's $300 \mathrm{~mm}$ drop tests. Of the four models that would have failed, two exceeded the IRB's maximum criterion of 550 $\mathrm{g}$, and two were below the minimum criterion of $200 \mathrm{~g}$. However, the two models with results below the IRB's $200 \mathrm{~g}$ criterion were most effective in attenuating impact energy and thus most likely to reduce concussion. The IRB's decision to impose a specification for headgear that places limits on its effectiveness in reducing concussion is in stark contrast with all other international helmet standards and the general philosophy underlying a large range of personal protective equipment and safety systems. To our knowledge, similar restrictions are not placed on headgear in rugby league and Australian rules football.

How do the test impacts reported here compare with head impacts in football? It is very difficult to measure or simulate the complex dynamics of these real life impact events because of energy attenuation by muscle, joint, or connective tissue forces applied through the neck to the head, and soft tissue deformation. However, on the basis of preliminary results of an unpublished study of rugby and Australian football head impacts resulting in concussion, the mean impact energy ranged between $45 \mathrm{~J}$ for non-loss of consciousness concussion and $75 \mathrm{~J}$ when loss of consciousness for longer than 1 minute was observed. Unlike the rigid headform-anvil impacts reported here, the real world impacts are between deformable objects.

In designing football headgear, it is important that safety benefits, which can be achieved by increasing the liner thickness and density, are considered in the context of the game and general-for example, weight and vision-and game specific-for example, scrums, rucks, and mauls - dimensional constraints. Increases 
in headgear thickness and density will increase its mass and therefore apply additional loads to the wearer's neck. Fortunately, performance improvements can be achieved with very minor design changes. The design of the best performing model, one that tends to be worn by rugby league players in Australia, was unique among those tested, as it consisted of a $12.5 \mathrm{~mm}$ thick foam layer over the lateral impact sites. Furthermore, the $12.5 \mathrm{~mm}$ comprised a $10 \mathrm{~mm}$ thick piece of medium density foam covered by a $2.5 \mathrm{~mm}$ thick piece of higher density foam. This provided consistently better protection for all test conditions, except centre front impacts, as the $2.5 \mathrm{~mm}$ insert did not extend to this part of the headgear.

A requirement that headgear should be designed to prevent only superficial injury is probably best considered from a fresh perspective, which requires an examination of current test methods and concerns about hard shell helmets. Headgear designed to prevent only lacerations and abrasions may consist of a thin (1-2 $\mathrm{mm}$ ) sheet of hard flexible plastic with thin comfort liners. The material could then be tested specifically for abrasion and penetration resistance. Such a design would offer no energy attenuation and the wearer would be unlikely to engage in reckless play as the impact forces applied to the head would be unchanged. It would also not reduce concussion. These comments highlight the point that clear choices need to be made about the function and role, if any, of headgear in football so that the most appropriate designs can be identified and considered by sporting bodies, associated organisations, and players.

In conclusion, the test results indicate that current commercially available football headgear is unlikely to reduce concussion or more severe head injury. Impact energy attenuation performance is poor compared with that of other types of helmets-for example, those designed for road users - and internationally recognised injury criteria. The absence of well conceived and internationally recognised standards governing soft headgear is a major deficiency in preventing neurological head injury.
The authors gratefully acknowledge the financial support of the New South Wales Sporting Injuries Committee. P R M is funded by the National Health and Medical Research Council of Australia. No commercial sponsorship was obtained for this study and no conflict of interest exists.

1 McCrory P. Neurological injuries in rugby and Australian rules football. In: Jordan B, Tsaris P, Warren R, eds. Sports neurology. 2nd ed. Philadelphia: Lippincott-Raven Publishers, 1998:441-9.

2 Newman J. Engineering considerations in the design of protective headgear. Proceedings of the American Association of Automotive Medicine 22nd Conference, Ann Arbor Michigan. Des Plaines, IL: Association of Advancement of Automotive Medicine, 1978:279-93.

3 Wilson BD. Protective headgear in rugby union. Sports Med 1998;25:333-7.

4 International Rugby Board. Laws of the game of rugby football. Dublin: IRB, 1996.

5 Gerrard DF, Waller AE, Bird YN. The New Zealand rugby injury and performance project: II. Previous injury experience of a rugby playing cohort. $\mathrm{Br} \quad \mathcal{F}$ Sports Med 1994;28:229-33.

6 Australia S. AS/NZS 2512.1.1:1999: methods of testing protective helmets, determination of impact energy attenuation: helmet drop test. Canberra, Australia: Standards Australia, 1999.

7 International Rugby Board. Standard performance specification for specific items of player's clothing, IRB Law 4M-98f. Dublin: IRB, 1998.

8 Hardy WN. Instrumentation in experimental design. In: Nahum AM, Melvin JW, eds. Accidental injury: biomechanics and prevention. New York: Springer, 1993:12-48.

9 Australia S. AS/NZS 2512.2:1998: methods of testing protective helmets. Method 2: general requirements for the conditioning and preparation of test specimens and laboratory conditions. Canberra: Standards Australia, 1998.

10 McCrory PR, Bladin PF, Berkovic SF. Retrospective study of concussive convulsions in elite Australian rules and rugby league footballers: phenomenology, aetiology, and outcome. BMF 1997;314:171-4.

11 McCrory P, Berkovic S. Videoanalysis of the motor and convulsive manifestations of concussion in acute spot related head injury. Neurology 2000;54:1488-91.

12 Ommaya A. Biomechanical aspects of head injuries in sports. In: Jordan B, Tsaris P, Warren R, eds. Sports neurology. Rockville, MD: Aspen Publishers Inc, 1990:84-98.

13 Ommaya AK, Gennarelli TA. Cerebral concussion and traumatic unconsciousness. Correlation of experimental and clinical observations of blunt head injuries. Brain 1974;97:633-54.

14 Gennarelli T, Thibault L, Graham D. Diffuse axonal injury: an important form of traumatic brain damage. Neuroscientist 1998;4:202-15.

15 Gennarelli TA. Mechanisms of brain injury. 7 Emerg Med 1993;11(suppl 1):5-11.

16 Ommaya A. Head injury mechanisms and the concept of preventative management: a review and critical synthesis. $\mathcal{F}$ Neurotrauma 1995;12:527-46.

17 McIntosh A, Kalleris D, Mattern R, et al. An evaluation of pedal cycle helmet performance requirements. In: 39th STAPP Car Crash Conference, 1995. Warrendale, PA: Society of Automotive Engineering, 1995:111-20.

18 McIntosh A, Kalleris D, Mattern R, et al. Head and neck injury resulting from low velocity direct impact. In: 37 th STAPP Car Crash Conference, 1993. Warrendale, PA: Society of Automotive Engineering, 1993:43-58. 